\title{
Synthesis and characterization of metastable, crystalline st12 germanium
}

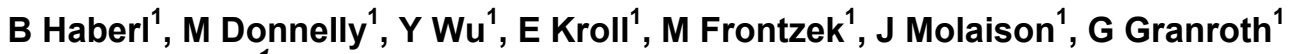 \\ ${ }^{1}$ Oak Ridge National Laboratory, Oak Ridge, TN \\ haberlb@ornl.gov
}

This work uses neutron scattering to characterize a metastable crystalline phase of germanium recovered from high pressure. Such metastable phases of silicon and germanium exhibit interesting functionality. They could potentially yield a Si or Ge structure with ideal band gap characteristics for solar power conversion, improved thin-film characteristics or - in the form of a hydride - even become a useful material for very high temperature superconductivity.

Several of such metastable, crystalline phases can be recovered from the metallic high-pressure polymorph of $\mathrm{Si}$ and Ge, the so-called $\beta$-Sn phase (I4[sub]1[/sub]/amd). This metallic polymorph forms upon room temperature compression to $\sim 11 \mathrm{GPa}$ from the standard diamond cubic $\mathrm{Si}$ or $\mathrm{Ge}(\mathrm{Fd}-3 \mathrm{~m})$. Upon decompression, this transition is not reversible and instead these metastable phase form. The exact crystal structure that is nucleated is thereby dependent on the exact decompression parameters such as temperature, rate or hydrostaticity. However, the need for synthesis pressures above $\sim 10 \mathrm{GPa}$ has typically limited the recoverable sample volumes. Hence, the majority of studies have been conducted computationally and fewer experimental characterizations have been performed. Thus, there are many open questions on the behavior and characteristics of these metastable phases.

Here, we focus on the simple tetragonal st12 structure of Ge (P4[sub]3[/sub]2[sub]1[/sub]2) by combining synthesis capabilities of the SNAP diffractometer of the Spallation Neutron Source with in situ high pressure diffraction on the WAND[sup]2[/sup] beamline of the High Flux Isotope Reactor and inelastic neutron scattering on recovered samples on the ARCS beamline of the Spallation Neutron Source. The st12 structure is synthesized using double-toroidal diamond anvils in a Paris-Edinburgh press from small pieces of a Ge wafer. The sample is pressurized to above $\sim 15 \mathrm{GPa}$ and kept at maximum pressure for several hours to ensure full conversion to the $\beta$ Sn phase.

The transition pathways is confirmed by in situ diffraction on WAND[sup]2[/sup]. Rietveld refinement of the Ge material under pressure confirms that all diamond-cubic material was indeed converted to the metallic $\beta$-Sn phase. It is noteworthy that this experiment represents the first use of the double-toroidal anvils on the WAND2 beamline and that pressures above $10 \mathrm{GPa}$ were achieved for the first time at the HFIR facility. Several such pellets were then measured on ARCS using incident energies of 30,50 , and $70 \mathrm{meV}$, which were combined to provide a fine resolution phonon density of states. The result closely matches the DFT predictions, although subtle differences may be detected.

Thus, in summary, these findings yield new insights into the potential use of the st12 phase as future semiconductor material and also open avenues for further characterization of such metastable phases of Si and Ge.

[b]Acknowledgments[/b]: This work was conducted at the SNAP, ARCS and WAND2 beamlines of the Spallation Neutron Source and the High Flux Isotope Reactor, respectively, both DoE Office of Science User Facilities operated by Oak Ridge National Laboratory.

Acta Cryst. (2020). A76, a131 